\title{
Avaliação dos níveis de ansiedade e seus fatores associados em estudantes internos de Medicina
}

\section{Evaluation of anxiety levels and their associated factors in medical interns}

\author{
Érika Guimarães Nogueira' (1) | erikagn20@hotmail.com \\ Nathália Camargo de Matos' (1) matos.nc@hotmail.com \\ Jordana Nascimento Machado' (1) jojo_26@hotmail.com \\ Luciana Benevides de Araújo' (1) lucianabenevides2@hotmail.com \\ Antonio Márcio Teodoro Cordeiro Silva' ${ }^{1}$ | marciocmed@gmail.com \\ \begin{tabular}{ll|l} 
Rogério José de Almeida' 1 | & rogerioufg@hotmail.com
\end{tabular}
}

\section{RESUMO}

Introdução: O período do internato para um estudante de Medicina é complexo e demanda atenção das escolas médicas em relação à saúde mental desses médicos em formação.

Objetivo: Este estudo teve como objetivo avaliar os fatores associados aos níveis de ansiedade em estudantes internos de Medicina.

Método: Trata-se de um estudo transversal analítico com abordagem quantitativa. Aplicaram-se dois questionários: um com dados sociodemográficos, pessoais e clínicos, e o Inventário de Ansiedade de Beck (BAI).

Resultados: Foram incluídos na pesquisa 140 estudantes internos de um curso de Medicina. A maioria tinha até 24 anos (67,9\%) e $70,7 \%$ eram do sexo feminino. Dos participantes, 54,2\% eram solteiros, 31\% eram adeptos de uma religião e 68,3\% moravam com familiares. Na comparação dos aspectos sociodemográficos com os níveis de ansiedade, identificou-se, no sexo feminino, uma frequência bem maior de ansiedade leve e moderada do que no sexo masculino $(p=0,0133)$. Aspectos pessoais e clínicos comparados com os níveis de ansiedade mostraram uma frequência maior de ansiedade em estudantes que afirmaram realizar terapia psiquiátrica ou psicológica $(p=0,0110)$. Ter insônia esteve relacionado com ansiedade de moderada a severa $(p<0,0001)$. A utilização de substâncias que alteram o sono foi associada com maior frequência a todos os níveis de ansiedade $(p=0,0099)$. A satisfação com o rendimento acadêmico teve menor relação com os níveis de ansiedade $(p=0,0017)$. Entretanto, maiores frequências de ansiedade de moderada a severa e ansiedade severa foram encontradas nos alunos que afirmaram ter pensado em abandonar o curso de Medicina $(p=0,0239)$.

Conclusão: O presente estudo revelou os aspectos sociodemográficos, pessoais e clínicos, e, consequentemente, os fatores de risco que estão mais associados ao nível de ansiedade em estudantes internos de Medicina. Ademais, expõem-se as consequências que os níveis de ansiedade podem provocar em um indivíduo, sendo imprescindível a adoção de medidas para combater e prevenir o desenvolvimento de sintomas ansiosos.

Palavras-chave: Estudantes de Medicina; Ansiedade; Internato Médico.

\section{ABSTRACT}

Introduction: The internship period for a medical student is complex and demands attention from medical schools regarding the mental health of these trainee doctors.

Objective: Accordingly, the aim of this study was to assess factors associated with anxiety levels among medical interns.

Method: A cross-sectional analytical study with a quantitative approach. Two questionnaires were applied, one with sociodemographic, personal and clinical data and the other consisting of the Beck Anxiety Inventory (BAl)

Results: 140 medical students were included in the research. Most of the students were 24 years old or under (67.9\%); 70.7\% were female. The majority were single (54.2\%), and lived with family members (68.3\%), while 31\% reported having a religion. When correlating sociodemographic aspects to levels of anxiety, a much higher frequency of females with mild and moderate anxiety was identified than that of males $(p=0.0133)$. Personal and clinical aspects compared to the levels of anxiety showed a higher frequency of anxiety in all levels of students who claimed to undergo psychiatric or psychological therapy $(p=0.0110)$. Suffering from insomnia is related to moderate to severe anxiety $(p<0.0001)$. Using substances that alter sleep is related to a higher frequency of students at all levels of anxiety $(p=0.0099)$. Satisfaction with academic performance is less related to anxiety levels $(p=0.0017)$. However, higher frequency in students with moderate to severe and severe anxiety were found in those who reported having already contemplated leaving medical school ( $p=0.0239)$.

Conclusion: This study revealed the sociodemographic, personal and clinical aspects and, consequently, the risk factors that are most frequently associated to anxiety in medical interns. Furthermore, it exposes the consequences that the variable levels of anxiety can have on an individual, and it is essential to adopt measures to combat and prevent the development of anxiety symptoms.

Keywords: Medical Students; Anxiety; Medical internship.

${ }^{1}$ Pontifícia Universidade Católica de Goiás, Goiânia, Goiás, Brasil.

Editora-chefe: Daniela Chiesa

Editor associado: Roberto Zonato Esteves

Recebido em 25/05/2020; Aceito em 01/12/20.

Avaliado pelo processo de double blind review. 


\section{INTRODUÇÃO}

A saúde mental dos estudantes universitários, principalmente os da área da saúde, sempre foi considerada como um sério motivo de preocupação, pois ela se constitui como um fator de risco para outros agravos relacionados à saúde física, tendo em vista a natureza muitas vezes estressante do ambiente acadêmico e do exercício profissional que pode levar ao desenvolvimento de diversos distúrbios emocionais ${ }^{1}$.

O estudante de Medicina está inserido em um novo contexto ao ingressar na vida acadêmica que pode alterar de forma drástica a sua saúde mental. Por conta disso, constante vigilância e apoio psicossocial são fundamentais ${ }^{2}$. Estudantes de medicina enfrentam um curso desgastante e vivenciam experiências estressantes. Durante o curso, expectativas e responsabilidades aumentam progressivamente, gerando tensões e angústias que afetam significantemente a saúde ${ }^{3}$.

O curso de Medicina, em especial o período do internato, requer do aluno uma jornada diária com alto grau de exigência. É necessário internalizar grande diversidade de conteúdos em pouco tempo, não somente referentes aos saberes médicos, mas também concernentes às habilidades que possibilitam as relações interpessoais, em que os alunos aprendem a lidar com sentimentos, sofrimentos e morte, envolvidos em contexto de muita competitividade, privação de lazer e insegurança técnica. A saúde mental talvez seja a parte mais afetada dos acadêmicos que vivenciam esse contexto inerente à formação médica ${ }^{3,4}$.

Em um estudo com estudantes de Medicina da cidade de Fortaleza, no Ceará, identificou-se que um dos principais aspectos relacionados à ansiedade nos estudantes de Medicina era a privação do sono, sendo essa uma das principais características referidas pelos alunos avaliados ${ }^{5}$. Outra contribuição para a ansiedade seria a presença de relacionamento insatisfatório com familiares, amigos, colega de sala e professores 5 .

A prevalência mundial de transtorno de ansiedade em 2015 estimada pela Organização Mundial da Saúde (OMS) foi de 3,6\%, o que corresponde a um número de 264 milhões de pessoas, refletindo um aumento de $14,9 \%$ em dez anos ${ }^{6}$. Os levantamentos feitos por região demonstraram que a América apresenta as maiores porcentagens tanto no sexo feminino quanto no masculino, sendo as do feminino ainda maiores. Sobre a prevalência por sexo, indivíduos do sexo feminino têm duas vezes mais probabilidade do que os do masculino, tendo o pico do diagnóstico na meia-idade ${ }^{6}$.

Nesse sentido, a análise do presente estudo se concentrou na avaliação da ansiedade em estudantes de Medicina do internato. Teve por objetivo identificar a prevalência e os fatores associados aos níveis de ansiedade em estudantes internos de Medicina.

\section{MÉTODOS}

Trata-se de um estudo transversal analítico com abordagem quantitativa. Esse método de pesquisa avalia o indivíduo e o fator de exposição simultaneamente, no mesmo intervalo de tempo analisado. Este estudo aborda uma situação em um momento não definido. Sendo assim, não há necessidade de mencionar o tempo de exposição a uma causa para gerar o efeito 7 .

A pesquisa foi realizada por meio de questionários aplicados a estudantes internos de Medicina da Pontifícia Universidade Católica de Goiás (PUC Goiás), que estavam cursando o internato, ou seja, entre o nono e décimo segundo período. Havia 198 estudantes regularmente matriculados em todo o internato. A princípio o objetivo era entrevistar todos os acadêmicos, mas, com as negativas durante a coleta de dados que ocorreu entre fevereiro e março de 2020, foi possível obter uma amostra de 140 internos (70,7\%).

Os critérios de inclusão foram: cursar Medicina na PUC Goiás, fazer parte do internato e estar presente no momento da aplicação do questionário. Os critérios de exclusão foram: estar de licença médica e apresentar os questionários respondidos de maneira incompleta.

Utilizaram-se dois questionários para a concretização dos objetivos desta pesquisa. O primeiro foi um questionário sociodemográfico, pessoal e clínico construído pelos pesquisadores com o intuito de obter dados de variáveis que possam se relacionar com a ansiedade.

O segundo questionário foi o Inventário de Ansiedade de Beck (Beck Anxiety Inventory - BAI) ${ }^{8}$, já adaptado e validado no Brasil9. Trata-se de uma escala de autorrelato que mede a intensidade dos sintomas da ansiedade. O BAl é constituído de 21 itens que são"afirmações descritivas da ansiedade", as quais devem ser avaliadas pelo indivíduo com referência a si mesmo em uma escala de quatro pontos que refletem níveis de gravidade crescente de cada sintoma: 1 = absolutamente não; 2 = levemente: não me incomodou muito; 3 = moderadamente: foi muito desagradável, mas pude suportar; 4 = gravemente: difícil de suportar.

A escala reflete manifestações somáticas, cognitivas e afetivas características da ansiedade e refere-se aos sintomas que incomodaram o indivíduo na última semana, podendo variar de 0 a 63 . O nível de ansiedade é classificado em ansiedade normal quando o escore total varia de 0 a 9 pontos, ansiedade de leve a moderada quando se obtém escore total entre 10 e 18 pontos, ansiedade de moderada a severa quando varia de 19 a 29 pontos e ansiedade severa entre 30 e 63 pontos $^{10}$.

Com os dados coletados, confeccionou-se um banco de dados utilizando o software Microsoft Excel. Posteriormente, realizou-se uma estatística descritiva com o cálculo de média, frequências absoluta e relativa percentual e desvio padrão.

$\mathrm{Na}$ sequência, utilizando do software BioEstat 5.3 foi 
aplicado o teste de $\mathrm{G}$ para testar a associação das variáveis sociodemográficas, pessoais e clínicas com os resultados do BAI, assumindo $p$-valor inferior ou igual a 0,05 como significativo.

Antes da coleta de dados, o presente trabalho foi encaminhado ao Comitê de Ética e Pesquisa (CEP) da PUC Goiás e aprovado com o Parecer n. 3.425.379. Os discentes que concordaram em participar da pesquisa assinaram o Termo de Consentimento Livre e Esclarecido.

\section{RESULTADOS}

Participaram da pesquisa 140 estudantes internos de um curso de Medicina. A idade média dos estudantes foi de $24,3( \pm 3,4)$, sendo $67,9 \%$ até 24 anos, com idade mínima de 21 e máxima de 49 anos. Do total, 70,7\% eram do sexo feminino, procedentes de Goiânia (56,3\%), que moravam com pai, mães ou irmãos (68,3\%). A maioria referiu estar solteira $(54,2 \%)$, ter fraco envolvimento religioso $(47,2 \%)$ e não trabalhar (62\%) (Tabela 1).

Em relação aos aspectos sociais e clínicos, 50\% dos estudantes referiram realizar atividade física frequentemente, $90,1 \%$ não apresentavam doenças crônicas, 38\% referiram ter doença psiquiátrica e $60,6 \%$ responderam estar fazendo terapia psiquiátrica ou psicológica. Em relação às horas dormidas, $81 \%$ responderam dormir menos de sete horas por noite, com 22,5\% tendo insônia, e 58,5\% faziam uso de substâncias que alteram o sono. A maioria dos estudantes $68,3 \%$ não estava satisfeita com o rendimento acadêmico e $45,1 \%$ já pensaram em abandonar o curso (Tabela 2 ).

Conforme Tabela 3, os resultados do BAI demonstraram que $52,9 \%$ tinham níveis de ansiedade normal; $25,7 \%$, de leve a moderada; $15 \%$, de moderada a severa; e 6,4\%, severa.

$\mathrm{Na}$ comparação dos aspectos sociodemográficos com os níveis de ansiedade, foram identificadas, no sexo feminino, maiores frequências em ansiedade leve e moderada do que no sexo masculino ( $p=0,0133$ ) (Tabela 4).

Na comparação dos aspectos pessoais e clínicos com os níveis de ansiedade, foi identificada uma frequência bem maior, em todos os níveis, de ansiedade nos estudantes que afirmaram realizar terapia psiquiátrica ou psicológica ( $p=0,0110)$. Ter insônia esteve relacionado com ansiedade de moderada a severa ( $p<0,0001$ ). A utilização de substâncias que alteram o sono esteve relacionada com maior frequência em todos os níveis de ansiedade $(p=0,0099)$. A satisfação com o rendimento acadêmico apresentou menor relação com os níveis de ansiedade ( $p=0,0017$ ). Entretanto, maiores frequências de ansiedade de moderada a severa e ansiedade severa foram encontradas nos alunos que afirmaram ter pensado em abandonar o curso de Medicina ( $p=0,0239)$ (Tabela 5).
Tabela 1. Caracterização dos aspectos sociodemográficos dos 140 estudantes internos de um curso de Medicina, Goiânia, Goiás, Brasil, 2020

\begin{tabular}{llll}
\hline \multicolumn{2}{c}{ Variáveis $(\mathbf{N}=\mathbf{1 4 0})$} & $\mathbf{n}$ & $\mathbf{f}(\%)$ \\
\hline Sexo & Masculino & 41 & \\
& Feminino & 99 & 70,7 \\
\hline \multicolumn{2}{l}{ Idade (anos) } & & \\
\multicolumn{2}{c}{ Até 24 anos } & 95 & 67,9 \\
\multicolumn{2}{c}{ Acima de 24 anos } & 45 & 32,1 \\
\hline
\end{tabular}

Procedência

\begin{tabular}{ccc} 
Goiânia & 80 & 56,3 \\
Interior de Goiás & 36 & 25,4 \\
Outro estado & 24 & 16,9 \\
\hline
\end{tabular}

Trabalha e cursa Medicina

$\begin{array}{lll}\text { Sim } & 52 & 36,6 \\ \text { Não } & 88 & 62,0\end{array}$

Período da Medicina

\begin{tabular}{cll} 
Período 9 & 27 & 19,0 \\
Período 10 & 31 & 21,8 \\
Período 11 & 37 & 26,1 \\
Período 12 & 45 & 31,7 \\
\hline
\end{tabular}

Atividades extracurriculares

$\begin{array}{lll}\text { Nenhuma } & 9 & 6,3\end{array}$

Uma Atividade $\quad 86 \quad 60,6$

Duas Atividades $\quad 38 \quad 26,8$

três ou mais atividades $\quad 7 \quad 4,9$

Estado civil

$\begin{array}{ccc}\text { Solteiro } & 77 & 54,2 \\ \text { Solteiro (namorando) } & 59 & 41,5 \\ \text { Casado } & 4 & 2,8\end{array}$

Envolvimento religioso

$\begin{array}{ccc}\text { Forte } & 44 & 31,0 \\ \text { Fraco } & 67 & 47,2 \\ \text { Nenhum } & 29 & 20,4\end{array}$

Mora com quem?

\begin{tabular}{ccc} 
Pai, mãe ou irmãos & 97 & 68,3 \\
Sozinho & 37 & 26,1 \\
Parentes & 6 & 4,2 \\
\hline
\end{tabular}


Tabela 2. Caracterização dos aspectos pessoais e clínicos dos 140 estudantes internos de um curso de Medicina, Goiânia, Goiás, Brasil, 2020

\begin{tabular}{ccc}
\hline Variáveis ( $\mathbf{N}=\mathbf{1 4 0})$ & $\mathbf{n}$ & $\mathbf{f}(\%)$ \\
\hline Frequência em bares e festas & & \\
Frequentemente & 49 & 34,5 \\
Às vezes & 65 & 45,8 \\
Raramente & 26 & 18,3 \\
\hline Frequência de atividade física & & \\
Frequentemente & 71 & 50,0 \\
Às vezes & 37 & 26,1 \\
Raramente & 32 & 22,5 \\
\hline Sim & 12 & 8,5 \\
Não & 128 & 90,1 \\
\hline Doença crônica & & \\
Sim & 52 & 36,6 \\
Não & 88 & 62,0 \\
\hline Doença psiquiátrica & & \\
Sim & 86 & 60,6 \\
Não & 54 & 38,0 \\
\hline Insônia & & \\
Sim & 32 & 22,5 \\
Não & 108 & 76,1 \\
\hline Terapia psiquiátrica/psicológica & &
\end{tabular}

Horas dormidas à noite

\begin{tabular}{lccc}
\multicolumn{2}{c}{$\begin{array}{c}\text { Menos de sete horas } \\
\text { Entre sete e nove horas }\end{array}$} & 115 & 81,0 \\
\hline Etilismo & & 25 & 17,6 \\
\cline { 3 - 4 } & Sim & 104 & 73,2 \\
& Não & 36 & 25,4 \\
\hline Tabagismo & & \\
& Sim & 9 & 6,3 \\
& Não & 131 & 92,3 \\
\hline
\end{tabular}

Usa substâncias que alteram o sono

$\begin{array}{lll}\text { Sim } & 83 & 58,5 \\ \text { Não } & 57 & 40,1\end{array}$

Satisfação com o rendimento acadêmico

$\begin{array}{lll}\text { Sim } & 43 & 30,3 \\ \text { Não } & 97 & 68,3\end{array}$

Pensou em abandonar o curso

\begin{tabular}{lll} 
Sim & 64 & 45,1 \\
Não & 76 & 53,5 \\
\hline
\end{tabular}

Tabela 3. Análise dos níveis de ansiedade dos 140 estudantes internos de um curso de Medicina, Goiânia, Goiás, Brasil, 2020

\begin{tabular}{lcc}
\hline \multicolumn{1}{c}{ Variáveis $(\mathbf{N}=140)$} & $\mathbf{n}$ & $\mathbf{f}(\%)$ \\
\hline $\begin{array}{l}\text { Níveis de ansiedade } \\
\text { Ansiedade normal (de 0 a 9 pontos) }\end{array}$ & 74 & 52,9 \\
\hline $\begin{array}{l}\text { Ansiedade de leve a moderada } \\
\text { (de 10 a 18 pontos) }\end{array}$ & 36 & 25,7 \\
\hline $\begin{array}{l}\text { Ansiedade de moderada a severa } \\
\text { (de 19 a 29 pontos) }\end{array}$ & 21 & 15,0 \\
\hline Ansiedade severa (de 30 a 63 pontos) & 9 & 6,4 \\
\hline
\end{tabular}

\section{DISCUSSÃO}

Os resultados deste estudo descreveram o perfil de estudantes internos de Medicina de uma instituição privada de ensino. Apesar de a pesquisa ter traçado o perfil sociodemográfico, clínico e pessoal de estudantes de uma única instituição e limitar-se a definir as características de discentes matriculados apenas nos dois últimos anos do curso, ela traz contribuições ao debate sobre a educação médica. Pela análise dos resultados, é possível perceber uma prevalência maior de discentes do sexo feminino $(70,7 \%)$, o que corrobora o padrão encontrado em outros dois estudos ${ }^{11,12}$ e contrasta com o resultado obtido por um estudo realizado na Universidade Federal do Pará (UFPA), no qual houve predomínio do sexo masculino $50,6 \%{ }^{13}$.

Quanto à faixa etária, este trabalho seguiu a tendência da média nacional de 24 anos de idade relatada nos estudos realizados em duas pesquisas ${ }^{14,15}$, sendo maior a prevalência de $67,9 \%$ de estudantes com até 24 anos. Há um rejuvenescimento da medicina no Brasil, com a média de idade dos profissionais diminuindo ao passar dos anos ${ }^{16}$.

Em relação ao trabalho remunerado, nossos resultados são semelhantes aos encontrados em outras pesquisas ${ }^{13,14}$, já que a maioria dos estudantes (62\%) afirmou que não trabalhava. Sabe-se que o baixo percentual de estudantes de Medicina que trabalham se opõe à realidade dos discentes de outros cursos universitários, em que a maioria faz estágios ou trabalha enquanto cursa a graduação.

Um estudo realizado com acadêmicos de Medicina da Universidade Federal do Amapá (UNIFAP) demostrou que 64,2\% dos estudantes moravam com familiares ${ }^{17}$, dado semelhante encontrado neste estudo que foi de $68,3 \%$. Outro estudo realizado conduzido na Universidade Federal do Rio Grande do Norte (UFRN) com 279 acadêmicos do curso de Medicina demonstrou que $64,9 \%$ dos estudantes moravam com os pais ${ }^{18}$. 
Tabela 4. Comparação dos aspectos sociodemográficos com os níveis de ansiedade dos 140 estudantes internos de um curso de Medicina, Goiânia, Goiás, Brasil, 2020.

\begin{tabular}{|c|c|c|c|c|c|c|c|c|c|}
\hline \multirow{3}{*}{ Variáveis $(N=140)$} & \multicolumn{8}{|c|}{ Inventário de Ansiedade de Beck (BAI) } & \multirow{3}{*}{ p-valor* } \\
\hline & \multicolumn{2}{|c|}{ Normal $(n=74)$} & \multicolumn{2}{|c|}{ Leve $(n=36)$} & \multicolumn{2}{|c|}{ Moderada $(n=21)$} & \multicolumn{2}{|c|}{ Severa $(n=9)$} & \\
\hline & $\mathbf{n}$ & $f(\%)$ & $\mathbf{n}$ & $f(\%)$ & $\mathbf{n}$ & $f(\%)$ & $\mathbf{n}$ & $f(\%)$ & \\
\hline \multicolumn{10}{|l|}{ Sexo } \\
\hline Masculino & 27 & 65,9 & 7 & 17,1 & 2 & 4,9 & 5 & 12,2 & \\
\hline Feminino & 47 & 47,5 & 29 & 29,3 & 19 & 19,2 & 4 & 4,0 & 0,0133 \\
\hline \multicolumn{10}{|l|}{ Idade (anos) } \\
\hline Até 24 anos & 51 & 53,7 & 24 & 25,3 & 14 & 14,7 & 6 & 6,3 & \\
\hline Acima de 24 anos & 23 & 51,1 & 12 & 26,7 & 7 & 15,6 & 3 & 6,7 & 0,9943 \\
\hline \multicolumn{10}{|l|}{ Procedência } \\
\hline Goiânia & 37 & 46,3 & 24 & 30,0 & 15 & 18,8 & 4 & 5,0 & \\
\hline Interior de Goiás & 23 & 63,9 & 7 & 19,4 & 3 & 8,3 & 3 & 8,3 & \\
\hline Outro estado & 14 & 58,3 & 5 & 20,8 & 3 & 12,5 & 2 & 8,3 & 0,5012 \\
\hline \multicolumn{10}{|l|}{ Trabalha e cursa medicina } \\
\hline Sim & 29 & 55,8 & 10 & 19,2 & 11 & 21,2 & 2 & 3,8 & \\
\hline Não & 45 & 51,1 & 26 & 29,5 & 10 & 11,4 & 7 & 8,0 & 0,2281 \\
\hline \multicolumn{10}{|l|}{ Período da Medicina } \\
\hline Período 9 & 15 & 55,6 & 6 & 22,2 & 6 & 22,2 & 0 & 0,0 & \\
\hline Período 10 & 15 & 48,4 & 7 & 22,6 & 4 & 12,9 & 5 & 16,1 & \\
\hline Período 11 & 23 & 62,2 & 8 & 21,6 & 4 & 10,8 & 2 & 5,4 & \\
\hline Período 12 & 21 & 46,7 & 15 & 33,3 & 7 & 15,6 & 2 & 4,4 & 0,3218 \\
\hline \multicolumn{10}{|l|}{ Atividades extracurriculares } \\
\hline Nenhuma & 4 & 44,4 & 3 & 33,3 & 0 & 0,0 & 2 & 22,2 & \\
\hline Uma atividade & 44 & 51,2 & 23 & 26,7 & 15 & 17,4 & 4 & 4,7 & \\
\hline Duas atividades & 19 & 50,0 & 10 & 26,3 & 6 & 15,8 & 3 & 7,9 & \\
\hline Três ou mais atividades & 7 & 100,0 & 0 & 0,0 & 0 & 0,0 & 0 & 0,0 & 0,1518 \\
\hline \multicolumn{10}{|l|}{ Estado civil } \\
\hline Solteiro & 40 & 51,9 & 20 & 26,0 & 13 & 16,9 & 4 & 5,2 & \\
\hline Solteiro (namorando) & 32 & 54,2 & 15 & 25,4 & 7 & 11,9 & 5 & 8,5 & \\
\hline Casado & 2 & 50,0 & 1 & 25,0 & 1 & 25,0 & 0 & 0,0 & 0,9532 \\
\hline \multicolumn{10}{|l|}{ Envolvimento religioso } \\
\hline Forte & 23 & 52,3 & 11 & 25,0 & 8 & 18,2 & 2 & 4,5 & \\
\hline Fraco & 34 & 50,7 & 21 & 31,3 & 7 & 10,4 & 5 & 7,5 & \\
\hline Nenhum & 17 & 58,6 & 4 & 13,8 & 6 & 20,7 & 2 & 6,9 & 0,5483 \\
\hline \multicolumn{10}{|l|}{ Mora com quem? } \\
\hline Pai, mãe ou irmãos & 50 & 51,5 & 26 & 26,8 & 14 & 14,4 & 7 & 7,2 & \\
\hline Sozinho & 23 & 62,2 & 6 & 16,2 & 6 & 16,2 & 2 & 5,4 & \\
\hline Parentes & 1 & 16,7 & 4 & 66,7 & 1 & 16,7 & 0 & 0,0 & 0,3334 \\
\hline *Teste G. & & & & & & & & & \\
\hline
\end{tabular}


Tabela 5. Comparação dos aspectos pessoais e clínicos com os níveis de ansiedade dos 140 estudantes internos de um curso de Medicina, Goiânia, Goiás, Brasil, 2020

\begin{tabular}{|c|c|c|c|c|c|c|c|c|c|}
\hline \multirow{3}{*}{ Variáveis $(N=140)$} & \multicolumn{8}{|c|}{ Inventário de Ansiedade de Beck (BAI) } & \multirow{3}{*}{ p-valor* } \\
\hline & \multicolumn{2}{|c|}{ Normal $(n=74)$} & \multicolumn{2}{|c|}{ Leve $(n=36)$} & \multicolumn{2}{|c|}{ Moderada $(n=21)$} & \multicolumn{2}{|c|}{ Severa $(n=9)$} & \\
\hline & $\mathrm{n}$ & $f(\%)$ & $\mathrm{n}$ & $f(\%)$ & $\mathbf{n}$ & $f(\%)$ & $\mathbf{n}$ & $f(\%)$ & \\
\hline \multicolumn{10}{|l|}{ Frequência em bares e festas } \\
\hline Frequentemente & 27 & 55,1 & 12 & 24,5 & 6 & 12,2 & 4 & 8,2 & \\
\hline Às vezes & 38 & 58,5 & 19 & 29,2 & 6 & 9,2 & 2 & 3,1 & \\
\hline Raramente & 9 & 34,6 & 5 & 19,2 & 9 & 34,6 & 3 & 11,5 & 0,0677 \\
\hline \multicolumn{10}{|l|}{ Frequência de atividade física } \\
\hline Frequentemente & 43 & 60,6 & 17 & 23,9 & 7 & 9,9 & 4 & 5,6 & \\
\hline Às vezes & 17 & 45,9 & 9 & 24,3 & 7 & 18,9 & 4 & 10,8 & \\
\hline Raramente & 14 & 43,8 & 10 & 31,3 & 7 & 21,9 & 1 & 3,1 & 0,4082 \\
\hline \multicolumn{10}{|l|}{ Doença crônica } \\
\hline Sim & 7 & 58,3 & 4 & 33,3 & 1 & 8,3 & 0 & 0,0 & \\
\hline Não & 67 & 52,3 & 32 & 25,0 & 20 & 15,6 & 9 & 7,0 & 0,5428 \\
\hline \multicolumn{10}{|l|}{ Doença psiquiátrica } \\
\hline Sim & 22 & 42,3 & 14 & 26,9 & 11 & 21,2 & 5 & 9,6 & \\
\hline Não & 52 & 59,1 & 22 & 25,0 & 10 & 11,4 & 4 & 4,5 & 0,1766 \\
\hline \multicolumn{10}{|l|}{ Terapia psiquiátrica/psicológica } \\
\hline Sim & 37 & 43,0 & 24 & 27,9 & 18 & 20,9 & 7 & 8,1 & \\
\hline Não & 37 & 68,5 & 12 & 22,2 & 3 & 5,6 & 2 & 3,7 & 0,0110 \\
\hline \multicolumn{10}{|l|}{ Insônia } \\
\hline Sim & 8 & 25,0 & 8 & 25,0 & 14 & 43,8 & 2 & 6,3 & \\
\hline Não & 66 & 61,1 & 28 & 25,9 & 7 & 6,5 & 7 & 6,5 & $<0,0001$ \\
\hline \multicolumn{10}{|l|}{ Horas dormidas à noite } \\
\hline Menos de sete horas & 55 & 47,8 & 33 & 28,7 & 19 & 16,5 & 8 & 7,0 & \\
\hline Entre sete e nove horas & 19 & 76,0 & 3 & 12,0 & 2 & 8,0 & 1 & 4,0 & 0,0885 \\
\hline \multicolumn{10}{|l|}{ Etilismo } \\
\hline Sim & 56 & 53,8 & 25 & 24,0 & 15 & 14,4 & 8 & 7,7 & \\
\hline Não & 18 & 50,0 & 11 & 30,6 & 6 & 16,7 & 1 & 2,8 & 0,6359 \\
\hline \multicolumn{10}{|l|}{ Tabagismo } \\
\hline Sim & 5 & 55,6 & 3 & 33,3 & 1 & 11,1 & 0 & 0,0 & \\
\hline Não & 69 & 52,7 & 33 & 25,2 & 20 & 15,3 & 9 & 6,9 & 0,7297 \\
\hline \multicolumn{10}{|l|}{ Usa substâncias que alteram o sono } \\
\hline Sim & 40 & 48,2 & 19 & 22,9 & 19 & 22,9 & 5 & 6,0 & \\
\hline Não & 34 & 59,6 & 17 & 29,8 & 2 & 3,5 & 4 & 7,0 & 0,0099 \\
\hline \multicolumn{10}{|c|}{ Satisfação com o rendimento acadêmico } \\
\hline Sim & 33 & 76,7 & 6 & 14,0 & 2 & 4,7 & 2 & 4,7 & \\
\hline Não & 41 & 42,3 & 30 & 30,9 & 19 & 19,6 & 7 & 7,2 & 0,0017 \\
\hline \multicolumn{10}{|l|}{ Pensou em abandonar o curso } \\
\hline Sim & 26 & 40,6 & 18 & 28,1 & 13 & 20,3 & 7 & 10,9 & \\
\hline Não & 48 & 63,2 & 18 & 23,7 & 8 & 10,5 & 2 & 2,6 & 0,0239 \\
\hline
\end{tabular}


No presente estudo, verificou-se uma alta prevalência de ansiedade da amostra estudada. Estudantes de Medicina são expostos a um ambiente emocionalmente exaustivo de treinamento e trabalho e a demandas acadêmicas estressantes. Consequentemente, psicopatologias são frequentes nessa população ${ }^{19}$. Níveis de sofrimento psíquico nesse grupo, particularmente a ansiedade patológica, são mais altos do que na população em geral ${ }^{20}$.

O sofrimento psicológico raramente é verbalizado pelos estudantes de Medicina, pois isso, em geral, está associado à fraqueza e é percebido como uma barreira à prática médica ${ }^{21}$. Como a ansiedade pode persistir após o término da faculdade médica, os jovens médicos estarão em condições abaixo do ideal para iniciar a carreira, o que poderá afetar a prestação de serviços e a qualidade do atendimento. Uma pesquisa sobre a saúde de estudantes de Medicina revelou que $68,2 \%$ dos participantes relataram ansiedade patológica ${ }^{22}$.

Em relação aos sintomas ansiosos, ser mulher foi associado com maior ansiedade. As mulheres apresentam maior prevalência de transtornos ansiosos ${ }^{23}$. Elas são mais propensas ao estresse e à ansiedade do que os homens, pois possuem uma maior carga de atividades e mais sintomas físicos. Isso decorre do fato de haver uma cobrança social dos múltiplos papéis a serem desempenhados pela mulher, o que está associado à necessidade de reafirmarem a sua competência em espaços liderados por homens. Além disso, há também as alterações hormonais próprias do sexo feminino que contribuem para esse perfil ${ }^{24}$.

As mulheres são consideradas mais conscientes dos seus sentimentos do que os homens e, dessa forma, demonstram com maior clareza seus sintomas. Pressupõe-se que os homens também tenham altos níveis de ansiedade, tanto quanto as mulheres, mas não relatam nos questionários pela falta de compreensão do que os angustia ${ }^{17}$.

Conforme os resultados apresentados, $60,6 \%$ dos entrevistados responderam que estavam fazendo terapia psiquiátrica ou psicológica, o que pode estar associado com maior nível de ansiedade. Entende-se que o motivo dessa relação seria o maior autoconhecimento e, consequentemente, a busca de ajuda profissional. Mesmo com os altos índices de sofrimento psicológico entre os estudantes de Medicina, estima-se que $8 \%$ a $15 \%$ buscam suporte psicológico. Trata-se de uma atitude prejudicial, já que pode gerar um maior risco de burnout, suicídio, entre outros, além de reduzir a capacidade de aprendizado e a qualidade no atendimento ao paciente ${ }^{25}$.

Entre os motivos que levam os acadêmicos a não procurar apoio psicológico, estão a alta carga horária exigida pelo curso - o que significa pouco tempo disponível para outras necessidades -, a sensação de que deveriam estar estudando em vez de procurarem atendimento, a falta de conhecimento acerca dos sintomas e o estigma que ainda existe quanto à procura de ajuda e ao tratamento de doenças mentais ${ }^{25}$.

Acredita-se que a solicitação de ajuda seria uma expressão de vulnerabilidade e que não seria aceitável dentro do campo médico, e, por isso, os discentes evitam assumir que necessitam de algum tipo de suporte. Há ainda os estudantes que compreendem as próprias angústias, porém, recorrem à automedicação, o que pode surtir prejuízos iguais ao não tratamento ou até piores com reações adversas significativas ${ }^{25}$.

Em relação ao sono, 22,5\% dos estudantes afirmaram sofrer de insônia, sendo esse fator relacionado com a ansiedade de moderada a grave. Existe uma importante relação entre a qualidade do sono e o estado emocional da pessoa. A ansiedade tem como consequência a insônia aguda ou crônica, que acarreta prejuízos físicos, cognitivos e emocionais, além de provocar o desequilíbrio do sistema imunológico. Durante o sono, há um descanso fisiológico, as células são renovadas e a memória é consolidada. Sendo assim, diante dessa falta de sono, a capacidade de concentração e de aprendizagem fica reduzida ${ }^{26}$.

Podem-se citar ainda uma maior dificuldade de tomar decisões e de elaborar hipóteses diagnósticas, uma lentificação para estabelecer a conduta, uma redução da qualidade da relação médico-paciente e um aumento da ansiedade, o que acaba por gerar um ciclo vicioso. Alguns fatores são claramente influenciadores da qualidade e quantidade do sono, entre os quais se destacam: novamente a carga horária alta, os plantões, as provas e a privação do sono para que se obtenha um maior rendimento acadêmico ${ }^{26}$.

Outro fator que influencia negativamente a qualidade de vida dos discentes está relacionado à utilização de substâncias que alteram o sono. Dos participantes desta pesquisa, 58,5\% utilizavam substância desse tipo, o que está relacionado com os maiores níveis de ansiedade. Há os estimulantes naturais, como a cafeína e a guaranina, e os sintéticos, como a taurina e o metilfenidato. Ambos estimulam o sistema nervoso central. Essas substâncias aumentam a concentração e fornecem mais energia, o que é extremamente vantajoso para quem está estudando. Porém, em altas doses, podem causar agitação, ansiedade, angústia, estresse e delírio. A cafeína, que é a mais utilizada, em doses acima de $600 \mathrm{mg}$ pode causar confusão mental e provocar erros em atividades intelectuais ${ }^{27}$. Os estudantes de Medicina são muito propensos ao consumo excessivo de psicoestimulantes por conta da privação de sono e da necessidade de maior concentração ${ }^{28}$.

Identificou-se que $68,3 \%$ dos discentes não estavam satisfeitos com o próprio rendimento acadêmico e 45,1\% já pensaram em abandonar o curso. Entre esses últimos, observou-se maior índice de ansiedade de moderada a grave. A ansiedade, como já mencionado, pode reduzir o índice de acerto 
dos estudantes, causar prejuízos ao aprendizado e culminar no abandono do curso ${ }^{29}$. O motivo pode ser explicado pela sintomatologia da ansiedade, que, quando muito acentuada, causa ameaça ao bem-estar físico e psicológico, com consequente dificuldade em manter um bom desempenho acadêmico ${ }^{30}$.

Por tratar-se de um fenômeno complexo, é necessário observar que há limitações, principalmente quando se estabelece associação entre as variáveis estudados e os níveis de ansiedade. Assim, é importante também pensar na hipótese de causalidade reversa, uma vez que os achados podem ter sua causa condicionada pela própria ansiedade.

\section{CONCLUSÃO}

No presente estudo, verificou-se que a maioria dos estudantes de medicina do internato apresentou níveis normais de ansiedade. Quando comparados aos aspectos sociodemográficos, observou-se que os níveis de ansiedade foram maiores no sexo feminino. Constataram-se maiores sintomas de ansiedade nos estudantes que faziam terapia, tinham insônia e utilizavam substâncias que alteram o sono.

Nestes trabalho, verificou-se que os estudantes que tinham maior satisfação com o rendimento acadêmico tiveram níveis menores de ansiedades, e aqueles que apresentaram ansiedade de moderada a severa pertenciam ao grupo de alunos que já pensaram em abandonar o curso.

Os estudantes de Medicina lidam com um curso que exige muito tanto física quanto emocionalmente. Trata-se de um curso de período integral, em que os erros não são aceitos, exigindo cada vez mais do acadêmico. Além disso, é um curso que trabalha com as dores e os sentimentos de uma pessoa, e, por conta disso, muitos discentes não conseguem lidar com esse contexto e passam a vivenciar com o paciente a dor dele. Por causa dessa grande exigência, muitos desses estudantes tendem a desenvolver diversos agravos à saúde mental, dentes estes, a ansiedade.

\section{CONTRIBUIÇÃO DOS AUTORES}

Todos os autores participaram igualmente da construção do artigo.

\section{CONFLITO DE INTERESSES}

Não há conflito de interesses neste estudo.

\section{FINANCIAMENTO}

Não houve financiamento para a realização desta pesquisa.

\section{REFERÊNCIAS}

1. Carvalho MCP, Junqueira LG, Cerdeira CD, Costa AMDD, Santos GB. Levantamento da situação de saúde mental e uso de ansiolíticos e antidepressivos por acadêmicos do curso de odontologia de uma universidade do sul de Minas Gerais. Revista da Universidade Vale do Rio Verde. 2017;15(1):489-96.

2. International Federation of Medical Students' Associations of Brazil. Saúde mental do estudante de medicina. Fortaleza; 2016 [acesso em $10 \mathrm{abr}$ 2020] Disponível em: http://ifmsa.net.br/wp-content/uploads/2017/02 / DP-Sau\%CC\%81de-Mental-do-Estudante-de-Medicina.pdf?07d318.

3. Tanaka MM, Furlan LL, Branco LM, Valerio NI. Adaptação de alunos de medicina em anos iniciais de formação. Rev Bras Educ Med. 2016;40(4):663-8.

4. Ferreira CMG, Kluthcovsky ACGC, CordeiroTMG. Prevalência de transtornos mentais comuns e fatores associados em estudantes de medicina: um estudo comparativo. Rev Bras Educ med. 2016;40(2):268-77.

5. Leão AM, Gomes IP, Ferreira MJM, Cavalcanti LPG. Prevalência e fatores associados à depressão e ansiedade entre estudantes universitários da área da saúde de um grande centro urbano do Nordeste do Brasil. Rev Bras Educ med. 2018;42(4):55-65.

6. World Health Organization. Depression and other common mental disorders: global health estimates. WHO; 2017 [acesso em 12 abr 2020] Disponível em: http://www.who.int/iris/handle/10665/254610.

7. Hochman B, Nahas FX, Oliveira RSF, Ferreira LM. Desenhos de pesquisa. Acta Cir Bras. 2005;20(2):2-9.

8. Beck AT, Epstein N, Brown GK, Steer RA. An inventory for measuring clinical anxiety: psychometric properties. J Consult Clin Psychol. 1988;56(6):893-7.

9. Cunha JA. Manual da versão em português das escalas de Beck. São Paulo: Casa do Psicólogo; 2001.

10. Creamer M, Foran J, Bell R. The Beck Anxiety Inventory in a non-clinical sample. Behav Res Ther. 1995;33(4):477-85.

11. Alvarenga VC, Coelho JLS, Rodrigues IC, Motta VMC, Silva LS. Perfil dos estudantes dos dois primeiros anos do curso de medicina de uma instituição privada de ensino de Minas Gerais. Rev Saúde Dinâmica. 2019;1(1):1-19.

12. Silva LS, Smaidi K, Pires MHR, Pires OC. Prevalência de dor crônica e fatores associados em estudantes de medicina. Rev Dor. 2017;18(2):108-11.

13. Rego RM, Marques NA, Monteiro PC, Barbosa CLO, Lins NAA, Caldas CAM $O$ perfil atual do estudante de medicina e sua repercussão na vivência do curso. Para Res Med J. 2018;2(1-4):e05.

14. Estrela YCA, Rezende ACC, Guedes AF, Pereira CO, Sousa MNA. Estresse e correlatos com características de saúde e sociodemográficas de estudantes de medicina. CES Med. 2018;32(3):215-25.

15. Toti TG, Bastos $F A$, Rodrigues $P$. Fatores associados à ansiedade e depressão em estudantes universitários do curso de educação física. Rev Saúde Física e Mental. 2018;6(2):1-10

16. Scheffer M, Cassenote A, Guilloux AGA, Biancarelli A, Miotto BA, Mainardi GM. Demografia médica no Brasil 2018. São Paulo: FMUSP, CFM, Cremesp; 2018 [acesso em 15 abr 2020]. Disponível em http://www.flip3d.com.br/ $\mathrm{web} / \mathrm{pub} / \mathrm{cfm} /$ index10/?numero=15\&edi cao=4278\#page/1.

17. Oliveira GS, Rocha CA, Santos BEF, Sena IS, Favaro L, Guerreiro MC Prevalência e fatores associados a depressão em estudantes de medicina da Universidade Federal do Amapá. Rev Med Saúde Brasília. 2017;5(3):186-99.

18. Costa DS, Medeiros NSB, Cordeiro RA, Frutuoso ES, Lopes JM, Moreira SNT. Sintomas de depressão, ansiedade e estresse em estudantes de Medicina e estratégias institucionais de enfrentamento. Rev Bras Educ Med. 2020;44(1):e040.

19. Baeza-Velasco C, Genty C, Jaussent I, Benramdane M, Courtet P, Olié E. Study protocol of a randomized multicenter controlled study of intervention based on attention versus relaxation to reduce emotional exhaustion in medical students in France: the "Prevent" study. BMC Psychiatry. 2020;20(1):115. 
20. Rotenstein LS, Ramos MA, Torre M, Sega JB, Peluso MJ, Guille CM, et al. Prevalence of depression, depressive symptoms and suicidal ideation among medical students: a systematic review and meta-analysis. JAMA. 2016;316(21):2214-36.

21. Kothari V, George N, Hamid O. Prestação de apoio à saúde mental para estudantes de medicina. Adv Med Educ Pract. 2018;9:925-6.

22. InterSyndicale Nationale Autonome Représentative des Internes de Médicine Générale. Enquête Santé Mentale Jeunes Médecins; 2017 [acesso em 12 abr 2020]. Disponível em: https://isni.fr/wp-content/ uploads/2020/02/enquetesantementale.pdf.

23. Puthran R, Zhang MW, Tam WW, Ho RC. Prevalence of depression amongst medical students: a meta-analysis. Med Educ. 2016;50(4):456-68.

24. Wahed WAY, Hassan SK. Prevalence and associated factors of stress, anxiety and depression among medical Fayoum University students. Alexandria J Med. 2017;53(1):77-84.

25. Roberto A, Almeida A. Saúde mental de estudantes de medicina: estudo exploratório da Universidade da Beira Interior. Acta Med Port. 2011;24(S2):279-86.
26. Andrade LP, Souza AP, Souza AFP, Batiston GT, Roque GPC, Silva JYF, et al. Ansiedade versus alteração do padrão de sono-vigília em estudantes de medicina. Rev Ensino, Educação e Ciências Humanas. 2017;18(3):232-8.

27. Silveira VI, Oliveira RJF, Caixeta MR, Andrade BBP, Costa RGL, Santos GB. Uso de psicoestimulantes por acadêmicos de medicina de uma universidade do sul de Minas Gerais. Revista da Universidade Vale do Rio Verde. 2015;13(2):186-92.

28. Morgan HL, Petry AF, Licks PAK, Ballester AO, Teixeira KN, Dumith SC. Consumo de estimulantes cerebrais por estudantes de medicina de uma universidade do extremo sul do Brasil: prevalência, motivação e efeitos percebidos. Rev Bras Educ Med. 2017;41(1):102-9.

29. Vasconcelos TC, Dias BRT, Andrade LR, Melo GF, Barbosa L, Souza E. Prevalência de sintomas de ansiedade e depressão em estudantes de medicina. Rev Bras Educ Med. 2015;39(1):135-42.

30. Menezes FMF, Rodrigues VLR, Barros LM, Almeida HMR, Lima PAL. Avaliação da ansiedade traço e estado em estudantes de Enfermagem, Medicina, Odontologia e Biomedicina. Semana de Pesquisa da Universidade Tiradentes- SEMPESq. 2018;18:1-3. 